\title{
Performance Analysis of Code-Multiplexed Transmitted-Reference Ultra-Wideband Systems
}

\author{
Mehmet Emin Tutay ${ }^{\sharp}$, Sinan Gezici ${ }^{\sharp}$, and H. Vincent Poor $^{\dagger}$ \\ $\sharp$ Dept. of Electrical and Electronics Engineering, Bilkent University, Ankara 06800, Turkey \\ $\dagger$ Department of Electrical Engineering, Princeton University, Princeton, NJ 08544, USA \\ \{tutay,gezici\}@ee.bilkent.edu.tr, poor@princeton.edu
}

\begin{abstract}
In code-multiplexed transmitted-reference (CMTR) ultra-wideband (UWB) systems, data signals and reference signals are transmitted using two distinct orthogonal codes. In this way, performance improvements and/or implementation advantages are obtained compared to transmitted-reference (TR) and frequency-shifted reference (FSR) ultra-wideband (UWB) systems. In this study, performance of CM-TR systems is investigated, and probability of error expressions are obtained. For the single user case, a closed-form expression for the exact probability of error is derived, whereas a Gaussian approximation, the accuracy of which depends on the number of frames per symbol, is considered for the multiuser case. Also, the maximum likelihood detector is derived, and numerical examples are presented.

Index Terms - Ultra-wideband, impulse radio, multiple-access interference, code-multiplexed transmitted-reference.
\end{abstract}

\section{INTRODUCTION}

Due to their large bandwidth and high time resolution, ultrawideband (UWB) signals can be used for high-speed data transmission [1], and accurate range and location estimation [2]. In addition, UWB systems can be used for low-to-medium data rate communication with low cost receivers. In order to implement UWB systems, impulse radio (IR) systems can be employed [3], [4]. In IR systems, a train of pulses with durations on the order of a nanosecond are transmitted. Each pulse resides in an interval called a frame, and a number of frames are employed for each information symbol. The information symbol can be carried by the positions or amplitudes of pulses. In multiple access environments, in order to prevent collisions and increase robustness against interfering users, pulses of each user are transmitted according to a timehopping $(\mathrm{TH})$ sequence, which aims to decrease the probability of collision between pulses of different users [3]. In addition to a data modulation scheme, each pulse can have a polarity randomization code that provides additional robustness against multiple access interference (MAI) and eliminates spectral lines that violate the UWB spectral mask [5].

In practice, each transmitted UWB pulse reaches a receiver via tens or even hundreds of paths in a multipath environment. Hence, to collect energy from multipath components, Rake receivers can be employed [6]. Due to the large number of fingers [7] and high sampling requirements, implementation of Rake receivers can be challenging for UWB systems. In order to ease the strict requirements of channel estimation, transmitted-reference (TR) UWB systems [8], [9] and frequency-shifted reference (FSR) UWB systems [10] have been proposed. In these types of systems, one reference pulse and one data pulse are sent in each frame. The reference pulse contains no information and its channel response is used at the receiver, removing the need for channel estimation. Despite this important advantage, TR and FSR UWB systems have some disadvantages and limitations. In TR systems, to achieve orthogonality between data and reference signals, an analog delay line, which creates miniaturization problems, is needed. In FSR systems the orthogonality is achieved in the frequency domain and the analog delay line requirement is removed, resulting in a significantly simpler receiver. However, this implementation has data rate limitations [11]. On the other hand, code-orthogonalized transmitted reference (COTR) or codemultiplexed transmitted-reference (CM-TR) UWB systems [12], [13], which employ two distinct orthogonal codes for orthogonalization purposes, require a much simpler receiver and have no data rate limitations.

Although CM-TR systems are investigated in [12]-[14] for single-user and multi-user systems, detailed probability of error analysis of such systems has not been performed before. In this study, CM-TR systems are investigated and exact and approximate expressions for error probabilities are obtained. In addition, instead of the conventional CM-TR receiver [12][14], a maximum likelihood (ML) receiver is proposed, and its performance is compared against the conventional receiver via simulations.

\section{Signal Model}

For a CM-TR UWB system, the transmitted signal corresponding to the $k$ th user is given by [11]

$$
s^{(k)}(t)=\sqrt{\frac{E_{k}}{2 N_{f}}} \sum_{j=0}^{N_{f}-1} a_{j}^{(k)}\left(1+b^{(k)} \tilde{d}_{j}^{(k)}\right) w\left(t-j T_{f}-c_{j}^{(k)} T_{c}\right)
$$

where $T_{f}$ and $T_{c}$ are, respectively, the frame and chip intervals, $N_{f}$ is the number of frames per symbol, $E_{k}$ is the symbol energy for user $k, w(t)$ is the UWB pulse with unit energy, $b^{(k)} \in\{-1,+1\}$ is the binary information symbol for user $k$, and $\tilde{d}_{j}^{(k)} \in\{-1,+1\}$ is the $j$ th element of the code that provides orthogonalization of the data bearing signal and the reference signal for the $k$ th user. In order to increase robustness against MAI and avoid spectral lines [5], polarity randomization codes $a_{j}^{(k)} \in\{-1,+1\}$, where $a_{j}^{(k)}$ and $a_{i}^{(l)}$ are independent for $(k, j) \neq(l, i)$, are also employed. In order to prevent catastrophic collisions between pulses of different users, a time-hopping code $c_{j}^{(k)} \in\left\{0,1, \ldots, N_{c}-1\right\}$ is assigned to each user, where $c_{j}^{(k)}$ and $c_{i}^{(l)}$ are independent for $(k, j) \neq(l, i)$.

In (1), two pulses can be considered in each frame: one multiplied with $a_{j}^{(k)}$ and the other with $a_{j}^{(k)} b^{(k)} \tilde{d}_{j}^{(k)}$. The first pulse is the reference pulse and the second one is the data (information bearing) pulse. In a CM-TR UWB system, the 
$\tilde{d}_{j}^{(k)}$, s are chosen in such a way that the sequence of reference pulses for each symbol is orthogonal to the that of the data pulses [11].

Suppose that the signal in (1) passes through an $L$-path channel. The channel impulse response can be written as

$$
h_{c}(t)=\sum_{l=1}^{L} \alpha_{l} \delta\left(t-\tau_{l}\right)
$$

where $\delta(t)$ is the Dirac delta function, and $\alpha_{l}$ and $\tau_{l}$ represent, respectively, the channel coefficient and the delay of the $l$ th path. Then, considering $K$ users and additive Gaussian noise, the received signal can be expressed as

$$
r(t)=\sum_{k=1}^{K} r_{k}(t)+n(t),
$$

where $n(t)$ is zero mean Gaussian noise with flat spectral density of $\sigma^{2}$ over the system bandwidth, and $r_{k}(t)$ is defined as the signal from the $k$ th user; that is,

$$
r_{k}(t)=\sqrt{\frac{E_{k}}{2 N_{f}}} \sum_{j=0}^{N_{f}-1} a_{j}^{(k)}\left(1+b^{(k)} \tilde{d}_{j}^{(k)}\right) \tilde{w}\left(t-j T_{f}-c_{j}^{(k)} T_{c}\right)
$$

with $\tilde{w}(t)=w(t) * h_{c}(t)$. Here $*$ denotes the convolution operator.

\section{RECEIVER STRUCTURE}

In order to estimate the transmitted information symbol corresponding to the $k$ th user, $b^{(k)}$, from the received signal in (1), the conventional receiver can be used [12], [13]:

$$
\hat{b}=\operatorname{sgn}\left\{\sum_{j=0}^{N_{f}-1} \tilde{d}_{j}^{(k)} \int_{j T_{f}}^{(j+1) T_{f}} r^{2}(t) d t\right\}
$$

where $\operatorname{sgn}\{\cdot\}$ denotes the sign of its argument.

Let $S_{k}$ and $\bar{S}_{k}$ represent the sets of frame indices for which $\tilde{d}_{j}^{(k)}=1$ and $\tilde{d}_{j}^{(k)}=-1$, respectively; i.e.,

$$
\begin{aligned}
& S_{k}=\left\{j \in \mathcal{F} \mid \tilde{d}_{j}^{(k)}=1\right\} \\
& \bar{S}_{k}=\left\{j \in \mathcal{F} \mid \tilde{d}_{j}^{(k)}=-1\right\}
\end{aligned}
$$

where $\mathcal{F}=\left\{0,1, \ldots, N_{f}-1\right\}$ is the set of frame indices [11]. In order to achieve orthogonality between reference and data signals, the following condition needs to be satisfied [11]:

$$
\left|S_{k}\right|=\left|\bar{S}_{k}\right|=N_{f} / 2 \text {. }
$$

From (4), it is observed that, for $b^{(k)}=1$, we transmit pulses in the frames indexed by $S_{k}$ and the frames indexed by $\bar{S}_{k}$ contain no pulses. Similarly, for $b^{(k)}=-1$, we transmit pulses in the frames indexed by $\bar{S}_{k}$ and the frames indexed by $S_{k}$ contain no pulses.

From (6) and (7), (5) can be expressed as

$$
\sum_{j \in S_{k}} \int_{\Gamma_{j}} r^{2}(t) d t \stackrel{\hat{b}^{(k)}=+1}{\underset{\hat{b}^{(k)}=-1}{<}} \sum_{j \in \bar{S}_{k}} \int_{\Gamma_{j}} r^{2}(t) d t
$$

which can be considered to be a generalized non-coherent detector for binary pulse position modulation (PPM) [11], [15],
[16], [17]. Note that, in (5), the integration over which the energy is calculated is taken as $T_{f}$, whereas a generic interval $\Gamma_{j}$ is used in (9). If the TH sequence for the user of interest is known, then the integration interval can be chosen in an optimal manner, as discussed in [18].

\section{Performance Analysis}

Without loss of generality, user 1 is considered to be the user of interest. Then, the expression in (9) can be written in terms of the difference of two terms as follows:

$$
D=\sum_{j \in S_{1}} \int_{\Gamma_{j}} r^{2}(t) d t-\sum_{j \in \bar{S}_{1}} \int_{\Gamma_{j}} r^{2}(t) d t \underset{\hat{b}^{(1)}=-1}{\stackrel{\hat{b}^{(1)}}{\gtrless}} 0+1
$$

Due to the presence of $K$ users in the system, the received signal $r(t)$ can be expressed as in (3) and (4). Let $\tilde{r}(t)=$ $\sum_{k=1}^{K} r_{k}(t)$ represent the sum of the received signals from all the users. Then, (10) can be written as

$$
\left.D=\sum_{j \in S_{\Gamma_{j}}} \int_{j}(\tilde{r}(t)+n(t))^{2} d t-\sum_{j \in \bar{S}_{\Gamma_{j}}} \int_{(\tilde{r}}(t)+n(t)\right)^{2} d t .
$$

Note that no subscripts are used with $S$ and $\bar{S}$ for convenience, and $S_{1}$ and $\bar{S}_{1}$ are implied unless stated otherwise.

Since $n(t)$ is zero mean Gaussian noise with a flat spectral density of $\sigma^{2}$ over the system bandwidth, the energy samples from the $j$ th frame $\int_{\Gamma_{j}}(\tilde{r}(t)+n(t))^{2} d t$ can be shown to be distributed as chi-square random variables [19]. It follows from the definition of the chi-square distribution that the sum of independent chi-square random variables is also chisquare distributed. Therefore, (11) can be represented as the difference of two chi-square random variables,

$$
D=D_{1}-D_{2}=\sum_{j \in S} \chi_{M}^{2}\left(\theta_{j}(\mathbf{b})\right)-\sum_{j \in \bar{S}} \chi_{M}^{2}\left(\theta_{j}(\mathbf{b})\right),
$$

where $\chi_{M}^{2}(\cdot)$ denotes a chi-square distributed random variable with $M$ degrees of freedom. $M$ represents the approximate dimensionality of the signal space, which is obtained from the time-bandwidth product, and $\theta_{j}(\mathbf{b})$ is the signal energy in the $j$ th frame (in the absence of noise) for a given set of binary information symbols b. From (3), (4) and (11), $\theta_{j}(\mathbf{b})$ can be obtained as

$$
\begin{aligned}
\theta_{j}(\mathbf{b}) & =\sum_{k_{1}=1}^{K} \sum_{k_{2}=1}^{K} \frac{\sqrt{E_{k_{1}} E_{k_{2}}}}{2 N_{f}} a_{j}^{\left(k_{1}\right)} a_{j}^{\left(k_{2}\right)}\left(1+b^{\left(k_{1}\right)} \tilde{d}_{j}^{\left(k_{1}\right)}\right) \\
& \times\left(1+b^{\left(k_{2}\right)} \tilde{d}_{j}^{\left(k_{2}\right)}\right) R_{\tilde{w}}^{j}\left(\left(c_{j}^{\left(k_{1}\right)}-c_{j}^{\left(k_{2}\right)}\right) T_{c}\right),
\end{aligned}
$$

where the last term $R_{\tilde{w}}^{j}(\cdot)$ can be considered as the correlation function between user $k_{1}$ and user $k_{2}$ in the $j$ th frame and is defined as

$$
\begin{aligned}
& R_{\tilde{w}}^{j}\left(\left(c_{j}^{\left(k_{1}\right)}-c_{j}^{\left(k_{2}\right)}\right) T_{c}\right) \\
& =\int_{\Gamma_{j}} \tilde{w}\left(t-j T_{f}-c_{j}^{\left(k_{1}\right)} T_{c}\right) \tilde{w}\left(t-j T_{f}-c_{j}^{\left(k_{2}\right)} T_{c}\right) d t .
\end{aligned}
$$




\section{A. Single User Case}

In the single user case, $\mathbf{b}=b^{(1)}$ and the probability of error can be expressed as

$P_{e}=\frac{1}{2} P\left\{D_{1}>D_{2} \mid b^{(1)}=-1\right\}+\frac{1}{2} P\left\{D_{1} \leq D_{2} \mid b^{(1)}=1\right\}$,

where $b^{(1)} \in\{-1,+1\}$ with equal probability and $D_{1}$ and $D_{2}$ are given as the two terms in (12) with $\tilde{r}(t)=r_{1}(t)$.

Note that, for $b^{(1)}=-1$, we transmit the pulses in the frames indexed by $\bar{S}$, and the other frames contain no pulses. Thus, using (13), one can obtain

$$
\theta_{j}(-1)= \begin{cases}0, & \text { if } j \in S \\ \theta, & \text { if } j \in \bar{S}\end{cases}
$$

where

$$
\theta \triangleq \frac{2 E_{1}}{N_{f}} \int_{\Gamma_{j}} \tilde{w}^{2}(t) d t=\frac{2 E_{1} E_{\tilde{w}}}{N_{f}} .
$$

Then, from (12), $D_{1}$ and $D_{2}$ are distributed as follows:

$$
D_{1} \sim \chi_{\frac{M N_{f}}{2}}^{2}(0) \text { and } D_{2} \sim \chi_{\frac{M N_{f}}{2}}^{2}\left(\theta N_{f} / 2\right) .
$$

Similarly, for $b^{(1)}=1$,

$$
D_{1} \sim \chi_{\frac{M N_{f}}{2}}^{2}\left(\theta N_{f} / 2\right) \text { and } D_{2} \sim \chi_{\frac{M N_{f}}{2}}^{2}(0) .
$$

From (18) and (19), the probability of error can be calculated as

$$
\begin{aligned}
P_{e} & =P\left\{D_{1}>D_{2} \mid b^{(1)}=-1\right\} \\
& =\int\left(1-\frac{\gamma\left(\frac{M N_{f}}{4}, \frac{x}{2 \sigma^{2}}\right)}{\Gamma\left(\frac{M N_{f}}{4}\right)}\right) \frac{1}{2 \sigma^{2}} \mathrm{e}^{-\left(x+\theta N_{f} / 2\right) / 2 \sigma^{2}} \\
& \times\left(\frac{x}{\theta N_{f} / 2}\right)^{\frac{M N_{f}}{8}-\frac{1}{2}} I_{\frac{M N_{f}}{4}-1}\left(\frac{\sqrt{x \theta N_{f} / 2}}{\sigma^{2}}\right) d x .
\end{aligned}
$$

Note that $P_{e}=P\left\{D_{1}>D_{2} \mid b^{(1)}=-1\right\}$ is used since $P\left\{D_{1}>D_{2} \mid b^{(1)}=-1\right\}=P\left\{D_{1} \leq D_{2} \mid b^{(1)}=1\right\}$ in (15) due to symmetry.

The probability of error expression in (20) is an accurate expression, which can be evaluated numerically, for example, using MATLAB. In order to illustrate the accuracy of this expression, we will compare it against a Gaussian approximation. To that aim, $D_{1}$ and $D_{2}$ can be approximated by Gaussian random variables with the following means and variances [18]:

$$
\begin{aligned}
& D_{1} \sim \mathcal{N}\left(\sigma^{2} \frac{N_{f} M}{2}, \sigma^{4} N_{f} M\right) \\
& D_{2} \sim \mathcal{N}\left(\sigma^{2} \frac{N_{f} M}{2}+\frac{\theta N_{f}}{2}, \sigma^{4} N_{f} M+2 \sigma^{2} \theta N_{f}\right)
\end{aligned}
$$

for $b^{(1)}=-1$, and

$$
\begin{aligned}
& D_{1} \sim \mathcal{N}\left(\sigma^{2} \frac{N_{f} M}{2}+\frac{\theta N_{f}}{2}, \sigma^{4} N_{f} M+2 \sigma^{2} \theta N_{f}\right) \\
& D_{2} \sim \mathcal{N}\left(\sigma^{2} \frac{N_{f} M}{2}, \sigma^{4} N_{f} M\right)
\end{aligned}
$$

for $b^{(1)}=1$. In addition, for a given binary information symbol

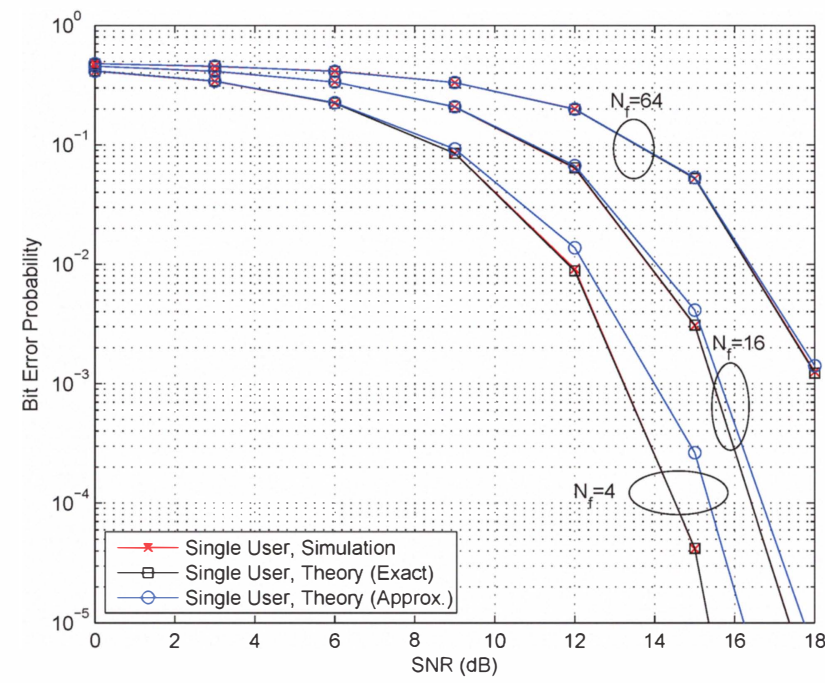

Fig. 1. BEP versus SNR for a single user system with $E_{1}=1$ and $N_{f}=4$, 16 , and 64 .

$b^{(1)}, D_{1}-D_{2}$ is also Gaussian as follows:

$$
D_{1}-D_{2} \sim \mathcal{N}\left(b^{(1)} \theta N_{f} / 2,2 \sigma^{4} N_{f} M+2 \sigma^{2} \theta N_{f}\right) .
$$

Thus, from (15), the probability of error can be expressed as

$$
P_{e} \approx Q\left(\frac{\theta N_{f} / 2}{\sqrt{2 \sigma^{2} N_{f}\left(M \sigma^{2}+\theta\right)}}\right),
$$

which can also be stated, based on (17), as

$$
P_{e} \approx Q\left(\frac{E_{1} E_{\tilde{w}}}{\sqrt{2 \sigma^{2}\left(N_{f} M \sigma^{2}+2 E_{1} E_{\tilde{w}}\right)}}\right) .
$$

In order to compare the expressions in (20) and (27), simulations and numerical evaluations have been performed. Fig. 1 plots the bit error probability (BEP) versus the signal-tonoise ratio (SNR) for different numbers of frames, $N_{f}$. From the figure, it is observed that, for a constant symbol energy, the performance of the receiver degrades as $N_{f}$ increases, which is expected from (27). In addition, there is good agreement between the exact theoretical results (obtained from (20)) and the simulation results, whereas the Gaussian approximation in (27) results in inaccurate error values for small values of $N_{f}$, as might be expected.

For the scenario in Fig. 1, a single path channel is considered for simplicity (realistic multipath channels are considered in Section VI) and the integration interval is taken as one pulse duration. Therefore, the degrees of freedom for the chi-square random variable in each frame is small since it is determined by the time duration and bandwidth product [11]. Therefore, the Gaussian approximation becomes accurate only for large $N_{f}$ values since the degrees of freedom of the decision variables are given by $M N_{f} / 2$ as shown in (18) and (19). In practical UWB channels, there can be a large number of multipath components; hence, a larger integration interval can be employed. Therefore, the Gaussian approximation may be accurate in practice.

\section{B. Multiuser Case}

In this section, the performance of the conventional receiver is analyzed in multiuser environments. Although it is difficult 
to obtain a reasonable expression for the exact probability of error in this case, a closed form expression can be obtained based on the Gaussian approximation similarly to that in [14].

Without loss of generality, user 1 is assumed to be the user of interest in a $K$-user system. Assuming equiprobable information symbols for all users, the probability of error can be expressed as

$$
\begin{aligned}
P_{e}=\frac{1}{2^{K}} & \sum_{\tilde{\mathbf{b}} \in\{ \pm 1\}^{K-1}}\left(P\left\{D_{1}-D_{2} \geq 0 \mid b^{(1)}=-1, \tilde{\mathbf{b}}\right\}\right. \\
& \left.+P\left\{D_{1}-D_{2}<0 \mid b^{(1)}=-1, \tilde{\mathbf{b}}\right\}\right)
\end{aligned}
$$

where $\tilde{\mathbf{b}}=\left[b^{(2)} \cdots b^{(K)}\right]^{T}$, and $D_{1}$ and $D_{2}$ are given by

$$
D_{1}=\sum_{j \in S} \chi_{M}^{2}\left(\theta_{j}(\mathbf{b})\right) \quad \text { and } \quad D_{2}=\sum_{j \in \bar{S}} \chi_{M}^{2}\left(\theta_{j}(\mathbf{b})\right) .
$$

In the following lemma, the asymptotic normality of $D_{1}$ and $D_{2}$ is stated.

Lemma 1: As $M N_{f} \rightarrow \infty, D_{1}$ and $D_{2}$ are Gaussian distributed as follows.

$$
\begin{aligned}
& D_{1} \sim \mathcal{N}\left(\sum_{j \in S}\left(\sigma^{2} M+\theta_{j}(\mathbf{b})\right), \sum_{j \in S}\left(2 M \sigma^{4}+4 \sigma^{2} \theta_{j}(\mathbf{b})\right)\right), \\
& D_{2} \sim \mathcal{N}\left(\sum_{j \in \bar{S}}\left(\sigma^{2} M+\theta_{j}(\mathbf{b})\right), \sum_{j \in \bar{S}}\left(2 M \sigma^{4}+4 \sigma^{2} \theta_{j}(\mathbf{b})\right)\right) .
\end{aligned}
$$

Proof: Please see [18]

Although Lemma 1 is in the form a Gaussian approximation, it is important to note that it differs from common Gaussian approximation approaches that are based on a large number of users. In this scenario, the Gaussian approximation becomes more accurate as $M N_{f}$ increases. In other words, even for a small number of users, the approximation is quite accurate for large $M N_{f}$.

Since $|S|=|\bar{S}|=N_{f} / 2$, and the difference of two independent Gaussian random variables is also Gaussian, the term $D_{1}-D_{2}$ is distributed as

$$
\mathcal{N}\left(\sum_{j \in S} \theta_{j}(\mathbf{b})-\sum_{j \in \bar{S}} \theta_{j}(\mathbf{b}), 2 \sigma^{4} M N_{f}+4 \sigma^{2} \sum_{j=0}^{N_{f}-1} \theta_{j}(\mathbf{b})\right) .
$$

Thus, the probability of error can be calculated from (28) as in (32), shown at the top of the next page.

Note that for the single user case, that is, $\mathbf{b}=b^{(1)}$, (32) reduces to (27) as expected. Although the expression in (32) can be difficult to evaluate for a large number of users, it can be used to obtain probability of error expressions for small numbers of users, which is in fact the case in many practical situations, such as indoor UWB applications [1].

\section{MAXimum-Likelihood (ML) Detector}

In order to provide a performance benchmark, the ML detector is investigated, and its exact and approximate calculations are discussed in this section. Although the ML receiver can be computationally complex in many cases, it is known to minimize the probability of error for equally likely symbols; hence, it serves as an optimal receiver.

In the ML detector, the set of information symbols $\mathbf{b}=$ $\left[b^{(1)} \ldots b^{(K)}\right]^{\mathrm{T}}$ is estimated as

$$
\hat{\mathbf{b}}=\arg \max _{\mathbf{b}} \log \left(p_{\mathbf{b}}(\mathbf{y})\right)=\arg \max _{\mathbf{b}} \sum_{j=0}^{N_{f}-1} \log \left(p_{\mathbf{b}}\left(y_{j}\right)\right),
$$

where $p_{\mathbf{b}}\left(y_{j}\right)$ is the probability density function (PDF) of a non-central chi-square random variable and is given by

$p_{\mathbf{b}}\left(y_{j}\right)=\frac{1}{2 \sigma^{2}}\left(\frac{y_{j}}{\theta_{j}(\mathbf{b})}\right)^{\frac{M}{4}-\frac{1}{2}} \mathrm{e}^{-\frac{\left(\theta_{j}(\mathbf{b})+y_{j}\right)}{2 \sigma^{2}}} I_{\frac{M}{2}-1}\left(\frac{\sqrt{\theta_{j}(\mathbf{b}) y_{j}}}{\sigma^{2}}\right)$

Note that for a given set of binary information symbols $b$, if the signal energy (in the absence of noise) is zero; that is, $\theta_{j}(\mathbf{b})=0$, then $y_{j}$ becomes central chi-square distributed.

It is also noted that the objective function above can be computationally complex to evaluate. Therefore, the Gaussian approximation can be used to provide a simpler alternative solution.

From Lemma 1, it is observed that the Gaussian approximation can be employed for large values of $M$. Hence, the PDF of $y_{j}$ can be written as

$$
p_{\mathbf{b}}\left(y_{j}\right)=\frac{1}{\sqrt{2 \pi} \sigma_{j}} \mathrm{e}^{-\frac{\left(y_{j}-\mu_{j}\right)^{2}}{2 \sigma_{j}^{2}}},
$$

where $\mu_{j}$ and $\sigma_{j}$ are given respectively by

$$
\begin{aligned}
\mu_{j} & =\sigma^{2} M+\theta_{j}(\mathbf{b}), \text { and } \\
\sigma_{j} & =2 M \sigma^{4}+4 \sigma^{2} \theta_{j}(\mathbf{b}) .
\end{aligned}
$$

Thus, (33) can be expressed alternatively as

$$
\begin{aligned}
& \arg \max _{\mathbf{b}} \log \left(p_{\mathbf{b}}(\mathbf{y})\right) \\
& =\arg \min _{\mathbf{b}} \sum_{j=0}^{N_{f}-1}\left\{\log \left(\sqrt{2 \pi} \sigma_{j}\right)+\frac{\left(y_{j}-\mu_{j}\right)^{2}}{2 \sigma_{j}^{2}}\right\} .
\end{aligned}
$$

Note that in order to implement this detector, the channel state information, the $\mathrm{TH}$ sequences, and the polarity and orthogonalization codes for all users must be known. Therefore, this detector can be considered only to provide a performance benchmark.

\section{Simulation Results and Conclusions}

In this section, further simulation results are presented in order to illustrate the results in the previous sections. The UWB pulse $w(t)$ is chosen as the second order derivative of the Gaussian pulse [2]; that is,

$$
w(t)=\left(1-\frac{4 \pi t^{2}}{\zeta^{2}}\right) \mathrm{e}^{-\frac{2 \pi t^{2}}{\zeta^{2}}} / \sqrt{E_{p}},
$$

where $E_{p}$ is a scalar chosen to set $w(t)$ to unit energy and $\zeta=T_{c} / 2.5$ determines the pulse width. The bandwidth of the receive filter is $5 \mathrm{GHz}$ and the channel statistics are taken from the IEEE 802.15.4a channel models CM1 and CM2 [20]. For the considered CM-TR UWB system, the system parameters 


$$
P_{e}=\frac{1}{2^{K}} \sum_{\tilde{\mathbf{b}} \in\{ \pm 1\}^{K-1}}\left\{Q\left(\frac{\sum_{j \in \bar{S}} \theta_{j}\left(\tilde{\mathbf{b}}, b^{(1)}=-1\right)-\sum_{j \in S} \theta_{j}\left(\tilde{\mathbf{b}}, b^{(1)}=-1\right)}{\sqrt{2 \sigma^{4} M N_{f}+4 \sigma^{2} \sum_{j=0}^{N_{f}-1} \theta_{j}\left(\tilde{\mathbf{b}}, b^{(1)}=-1\right)}}\right)+Q\left(\frac{\sum_{j \in S} \theta_{j}\left(\tilde{\mathbf{b}}, b^{(1)}=1\right)-\sum_{j \in \bar{S}} \theta_{j}\left(\tilde{\mathbf{b}}, b^{(1)}=1\right)}{\sqrt{2 \sigma^{4} M N_{f}+4 \sigma^{2} \sum_{j=0}^{N_{f}-1} \theta_{j}\left(\tilde{\mathbf{b}}, b^{(1)}=1\right)}}\right)\right\}
$$

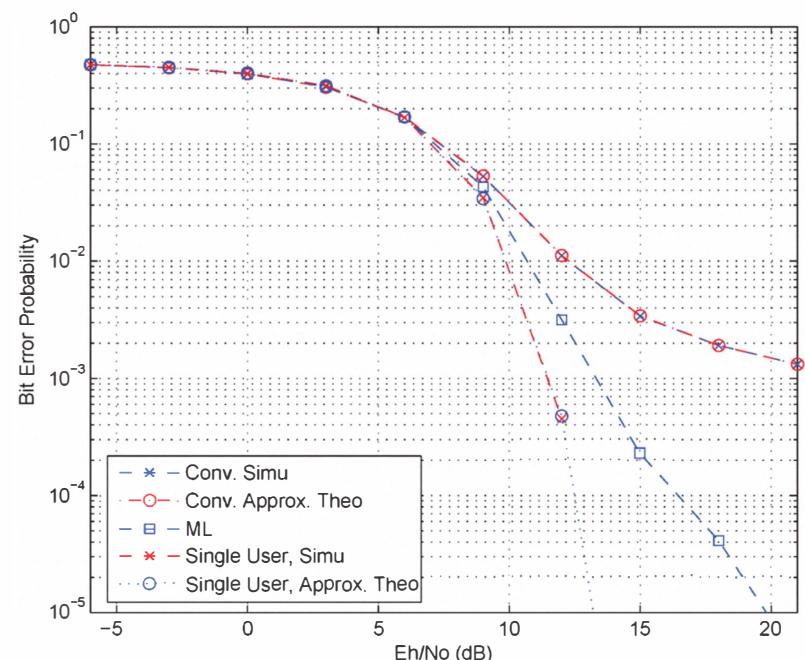

Fig. 2. BEP versus $E_{h} / N_{0}$ for a 2-user system for CM1 with $N_{f}=4$, $N_{c}=250, E_{1}=1$, and $E_{2}=1$.

are chosen as $N_{f}=4$ and $N_{c}=250$, which correspond to a data rate of $R_{b}=1 \mathrm{Mbit} / \mathrm{s}$ data rate.

Fig. 2 and Fig. 3 plot the BEPs for CM1 and CM2 for a two-user CM-TR UWB system. The BEPs are obtained as a function of the SNR defined in terms of $E_{h} / N_{0}$, where $E_{h}$ is the energy of $h(t)$ given by $E_{h}=\int_{\Gamma} h^{2}(t) d t\left(\Gamma_{i}=\Gamma, \forall i\right)$, with $h(t)=\sqrt{E_{1} /\left(2 N_{f}\right)} \tilde{w}(t)$ and $\tilde{w}(t)$ being the channel response to the unit energy pulse $w(t)$ in (39). In the figures, the receiver analyzed in Section IV is denoted as conventional ('Conv.'), and also the error probabilities in the absence of interference users ('Single User') are shown for comparison. In addition, the performance of the ML detector based on (38) is also illustrated.

From the plots, good agreement is observed between the theoretical and the simulation results for both single-user and two-user cases. Also, the conventional receiver has significantly worse performance than the ML receiver. However, it has implementation advantages over the ML receiver as discussed previously.

\section{REFERENCES}

[1] $\mathrm{H}$ Arslan, $\mathrm{Z}$ N Chen, and $\mathrm{M}-\mathrm{G}$. Di Benedetto (editors), Ultra Wideband Wireless Communications, Wiley-Interscience, Oct. 2006.

[2] Z. Sahinoglu, S. Gezici, and I. Guvenc, Ultra-wideband Positioning Systems: Theoretical Limits, Ranging Algorithms, and Protocols, Cambridge University Press, 2008.

[3] M. Z. Win and R. A. Scholtz, "Impulse radio: How it works," IEEE Communications Letters, vol. 2, pp. 36-38, Feb. 1998.

[4] D. Cassioli, M. Z. Win, and A. F. Molisch, "The ultra-wide bandwidth indoor channel: from statistical model to simulations," IEEE Journal on Selected Areas in Communications, vol. 20, pp. 1247-1257, Aug. 2002.

[5] Y.-P. Nakache and A. F. Molisch, "Spectral shape of UWB signals - influence of modulation format, multiple access scheme and pulse shape," Proc. IEEE Vehicular Technology Conference, vol. 4, pp. 25102514, Apr. 2003

[6] S. Gezici, M. Chiang, H. Vincent Poor, and H. Kobayashi, "Optimal and suboptimal finger selection algorithms for MMSE RAKE receivers in impulse radio ultra-wideband systems," Proc. IEEE Wireless Communications and Networking Conference, vol. 2 pp. 861-866, March 2005.

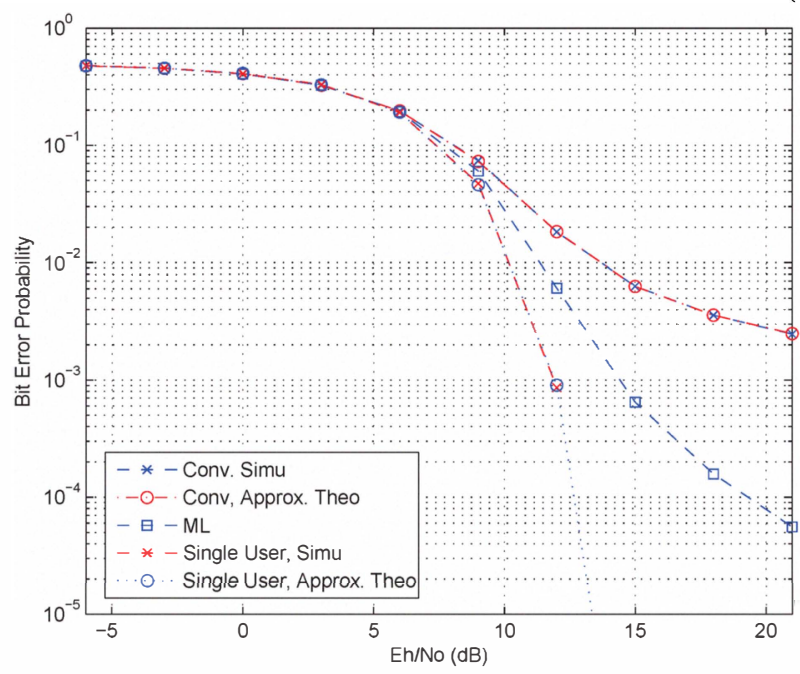

Fig. 3. BEP versus $E_{h} / N_{0}$ for a 2-user system for CM2 with $N_{f}=4$, $N_{c}=250, E_{1}=1$, and $E_{2}=1$

[7] M. Z. Win and R. A. Scholtz, "On the energy capture of ultrawide bandwidth signals in dense multipath environments," IEEE Communications Letters, vol. 2 pp. 245-247, Sept. 1998.

[8] R. Hoctor and H. Tomlinson, "Delay-hopped transmitted-reference RF communications," Proc. IEEE Conference on Ultra Wideband Systems and Technologies, pp. 265-269, May. 2002.

[9] J. D. Choi and W. E. Stark, "Performance of ultra-wideband communications with suboptimal receivers in multipath channels," IEEE Journal on Selected Areas in Communications, vol. 20 pp. 1754-1766, Dec. 2002.

[10] D. L. Goeckel and Q. Zhang, "Slightly frequency-shifted reference ultrawideband (UWB) radio," IEEE Transactions on Communications, vol. 55 pp. 508-519, March 2007.

[11] S. Gezici, "Coded-reference ultra-wideband systems," Proc. IEEE International Conf. on Ultra-Wideband, vol. 3, pp. 117-120, Sept. 2008.

[12] J. Zhang, H.-Y. Hu, L.-K Liu, and T.-F. Li, "Code-orthogonalized transmitted-reference ultra-wideband (UWB) wireless communication system," Proc. International Conference on Wireless Communications, Networking and Mobile Computing, pp. 528-532, Sept. 2007.

[13] A. A. D'Amico and U. Mengali "Code-multiplexed UWB transmittedreference radio," IEEE Transactions on Communications, vol. $56 \mathrm{pp}$. 2125-2132, Dec. 2008.

[14] A. A. D'Amico and U. Mengali, "Code-multiplexed transmittedreference UWB systems in a multi-user environment," IEEE Transactions on Communications, vol. 58, no. 3, pp. 966-974, March 2010.

[15] N. Song, M. Wolf, and M. Haardt, "Performance of PPM-based noncoherent impulse radio UWB systems using sparse codes in the presence of multi-user interference," in Proc. IEEE Wireless Communications and Networking Conference (WCNC 2009), Budapest, Hungary, April 2009.

[16] S. H. Song and Q. T. Zhang, "TH-CDMA-PPM with noncoherent detection for low rate WPAN, IEEE Transactions on Wireless Communications, vol. 7, no. 2, pp. 446-451, Feb. 2008.

[17] B. Hu and N. C. Beaulieu, "Exact bit error rate analysis of TH-PPM UWB systems in the presence of multiple-access interference," IEEE Commun. Lett., vol. 7, no. 12, pp. 572-574, Dec. 2003.

[18] M. E. Tutay, "Receiver design and performance analysis for code-multiplexed transmitted-reference ultra-wideband systems," M.Sc. Thesis, Bilkent University, Aug. 2010. [Available: www.ee.bilkent.edu.tr/ gezici/thesisMET.pdf]

[19] P. A. Humblet and M. Azizoğlu, "On the bit error rate of lightwave systems with optical amplifiers," Journal of Lightwave Technology, vol. 9, pp. 1576-1582, Nov. 1991.

[20] A. F. Molisch, et. al., "802.15.4a channel model - Final report," 2005 